\title{
Imanes permanentes: características, aplicaciones y futuro
}

\author{
Germán Antonio Pérez-Alcázar \\ Departamento de Física, Universidad del Valle, Cali, Colombia
}

\begin{abstract}
Resumen
Actualmente uno de los materiales magnéticos más utilizados en aplicaciones tecnológicas, son los imanes permanentes (IP) o materiales magnéticamente duros. En vista de su importancia, presento en este trabajo una revisión del estado del arte sobre estos materiales, partiendo de una revisión teórica de los conceptos y características más importantes que deben tenerse en cuenta para entender cómo funcionan; pasando por exponer y discutir los diferentes tipos de materiales que se utilizan en su fabricación y sus aplicaciones industriales relevantes; y finalmente discutir las rutas investigativas actuales y futuras en estos materiales, con el fin de obtener materiales para IP con mejores rendimientos y con menores costos. (c) Acad. Colomb. Cienc. Ex. Fis. Nat. 2016.
\end{abstract}

Palabras clave: imanes permanentes, sistemas nano-estructurados, anisotropía, coercitividad.

Permanent magnets: features, applications and future

\begin{abstract}
Actually, one of the most used magnetic materials in technological applications are the permanent magnets (PM) or hard magnetic materials. Due their importance, in this work I present a review of the state of the art about these materials; starting with a theoretical revision of the principal concepts and most important characteristics that are necessary to understand how they work; passing by the exposition and discussion of the different types of materials which are used for their production, and their relevant industrial applications; and finally to discuss the actual and future research pathways about these materials. This in order to obtain PM materials with better performance and low costs. (C) Acad. Colomb. Cienc. Ex. Fis. Nat. 2016.
\end{abstract}

Key words: Permanent magnets, nanostructured systems, anisotropy, coercivity.

\section{Introducción}

Los imanes permanentes (IPs) son materiales que, después de ser magnetizados por un campo magnético alto, mantienen su alta magnetización aun con cambios de temperatura o con la presencia de campos magnéticos externos (B. D. Cullity \& C. D. Graham (2009). A lo largo de la historia IPs han sido encontrados en forma natural, tal como la magnetita (Oxido de Fe), o han sido producidos por el hombre, tales como los alnico (IPs producidos principalmente con base a $\mathrm{Fe}, \mathrm{Al}$, Ni y Co), o las ferritas (ferrita de Ba y la de Sr) (B. D. Cullity \& C. D. Graham (2009) y R. C. O'Handley (2000)). A partir de la década de los 60 aparecieron los IPs con tierras raras, tales como sistemas con base a SmCo o YCo, y $\mathrm{Nd}_{2} \mathrm{Fe}_{14} \mathrm{~B}, \mathrm{La}_{2} \mathrm{Fe}_{14} \mathrm{~B}$ o $\mathrm{Pr}_{2} \mathrm{Fe}_{14} \mathrm{~B}$ (R. C. O'Handley). Más recientemente se han obtenido IPs libres de tierras raras obtenidos por aleación de diferentes elementos de transición tales como FePt, CoPt, MnAl y MnBi las cuales tienen estructura tetragonal (Felix Jimenez-Villacorta \& Laura H. Lewis (2014)).

El desarrollo de los IPs se da en vista de la necesidad de obtener gran cantidad de energía magnética almacenada en pequeños volúmenes, para ser incorporados en una enorme variedad de aplicaciones, que incluyen computadores y productos de consumo en oficinas, en la industria automotriz y de transporte, la electrónica, productos electrónicos caseros, productos para la automatización en fábricas, industria médica, industrias alternativas, y aplicaciones militares [USGS Mineral resources program, http://minerals.usgs.gov/. y Laura H. Lewis \& Félix Jiménez-Villacorta (2011)).

En este artículo de revisión se abordaran primero las ideas físicas importantes para entender el funcionamiento de un IP, se hará un recorrido histórico de los diferentes materiales y tipos de IPs, se detallaran las principales aplicaciones, y finalmente se hablará de las principales tendencias actuales y futuras en la obtención de IPs con mejores rendimientos.

\section{Conceptos básicos y terminología}

\section{a. Ciclo de histéresis}

Una manera de caracterizar los materiales magnéticos, es realizar un ciclo de histéresis, o sea una curva de

\footnotetext{
Correspondencia:

Germán Antonio Pérez Alcázar, gpgeperez@gmail.com

Recibido: 4 de mayo de 2016
}

Aceptado: 7 de junio de 2016 
magnetización $(M)$ o inducción magnética $(B)$ en función de un campo magnético aplicado $(H)$. Estas variables se relacionan mediante la expresión

$$
4 \pi M=B-H \text { (cgs) o } \mu_{0} M=B-\mu_{0} H(\mathrm{SI})
$$

Una curva típica para un material magnético se ilustra en la Figura 1, los números se utilizan para ilustrar la estructura de los dominios magnéticos en el ciclo de histéresis.

La notación cgs indica que las unidades utilizadas son centímetros, gramos y segundos, y la SI indica que se trabaja en el Sistema Internacional en metros, kilogramos y segundos. En la Figura 1 se muestra el ciclo obtenido de $B$ vs. $H$ o $M$ vs. $H$ y también se representa el comportamiento de la estructura de dominios. En la curva de magnetización inicial (a trazos) la estructura de dominios inicial (1) muestra como ellos están orientados en diferentes direcciones (o direcciones de fácil magnetización), esta estructura de dominios cambia con el aumento del campo. Los dominios con orientación cercana al campo crecen, y aquellos con orientación contraria al campo decrecen (2). Para un cierto campo se alcanza la magnetización de saturación $M_{\mathrm{s}}$, donde todos los dominios se orientan en la dirección del campo y se tiene un monodominio (3). Para este campo aplicado la inducción magnética tiene un valor $B_{s}$ dado por $B_{s}$ $=4 \pi M_{s}+H$ (cgs) o $B_{s}=\mu_{o}\left(M_{s}+H\right)$ (SI). Después de la saturación, se lleva $H$ a 0 , y este punto se conoce como $B_{r}$ o inducción magnética remanente (4), al cual le corresponde una magnetización remanente, $M_{r}$. Si el campo $H$ aumenta en dirección contraria se llega al campo coercitivo, $-H_{c}$, o coercitividad, que es el campo para el cual $B$ y $M$ son cero

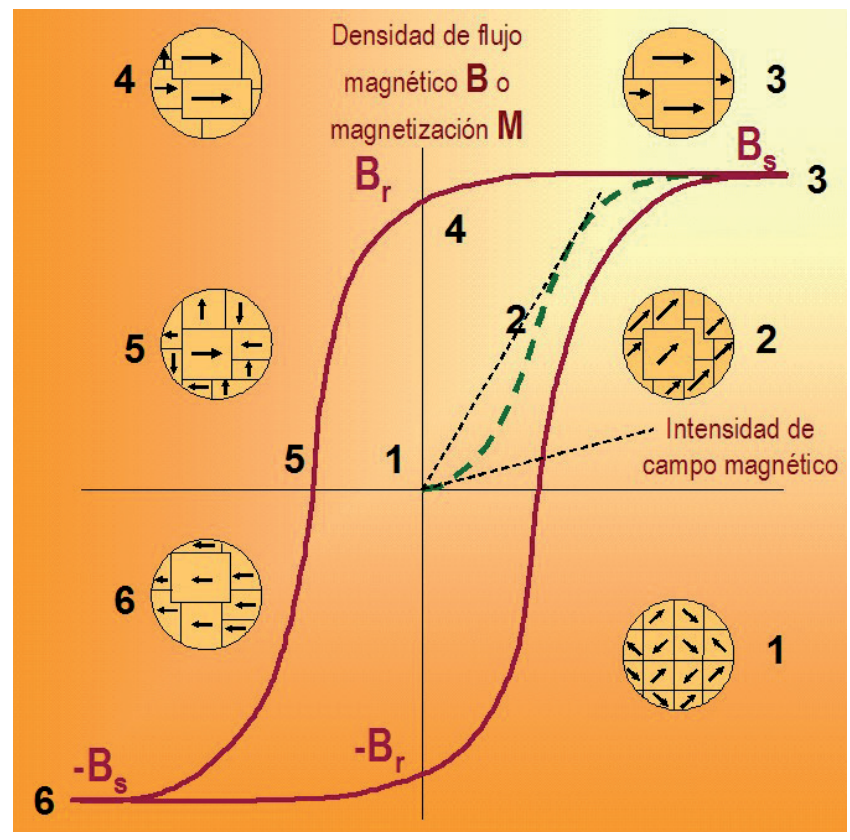

Figura 1. Ciclo de histéresis, $B$ vs. $H$ o $M$ vs. $H$ y algunas estructuras de dominios (https://www.google.com.co/search?ciclos +de+histéresis).
(5). En este punto los dominios se orientan de tal manera que la magnetización total es cero. Si el campo sigue aumentando se llegará a la saturación negativa con valores $-B_{s} \mathrm{o}-M_{s}(6)$. Si el módulo del campo se reduce se recorrerá una curva simétrica a la anterior, pasando por $-B_{r} \mathrm{o}-M_{r}$ cuando $H=0$, por $H_{c}$ y finalmente de nuevo a la saturación positiva.

Sin embargo los ciclos de $B$ vs. $H$ y de $M$ vs. $H$ no son idénticos, como lo muestra la Figura 1S, http://www.raccefyn. co/index.php/raccefyn/article/downloadSuppFile/361/1515, en el sistema cgs.

En cgs la curva de $B$ vs. $H$ se describe por la relación $B=$ $4 \pi M+H$, luego cuando $M$ satura la inducción tiene un valor $B_{\mathrm{s}}=4 \pi M_{\mathrm{s}}+H$. Cuando $H$ es cero se llega a la remanencia donde $B_{r}=4 \pi M_{r}$. Por este motivo la curva $M$ vs. $H$ se cambia por la de $4 \pi M$ vs. $H$, y de esta manera $B, 4 \pi M$ y $H$ tienen las mismas unidades. Las unidades de $B$ son gauss, las de $H$ son Oersted y las de $M$ son erg/Oe. $\mathrm{cm}^{3}$ o emu/ $\mathrm{cm}^{3}$, luego $4 \pi M$ debe estar dado en Oe o G. Cuando el campo aplicado $H$ se invierte hasta cuando $B=0$ se llega a un valor $-H_{c}$, pero $M$ es aún positivo y toma el valor $\left|H_{c} / 4 \pi\right|$. Para que M llegue a cero se necesita un campo llamado coercitividad intrínseca $H_{c i}$. La diferencia entre $H_{c}$ y $H_{c i}$ se nota claramente en la Figura 1S.

En el SI la curva de $B$ vs. $H$ se describe por la relación $B$ $=\mu_{0}(M+H)$, luego cuando $M$ satura la inducción tiene un valor $B_{s}=\mu_{0}\left(M_{s}+H\right)$. Cuando $H$ es cero se llega a la remanencia donde $B_{r}=\mu_{0} M_{r}$. Por este motivo en el SI la curva $M$ vs. $H$ se cambia por la de $\mu_{0} M$ vs. $H$, y de esta manera $B, \mu_{0} M$ y $\mu_{0} H$ tienen las mismas unidades. Las unidades de $B$ son tesla, las de $H$ y $M$ son $\mathrm{A} / \mathrm{m}$, luego $\mu_{0} M$ y $\mu_{0} H$ deben estar dados en tesla. Cuando el campo aplicado $H$ se invierte hasta cuando $B=0$ se llega a un valor $-H_{c}$, pero $M$ no es cero y toma el valor $H_{c}$. Para que M llegue a cero se necesita un campo llamado coercitividad intrínseca $H_{c i}$.

Dependiendo del valor del campo coercitivo los materiales magnéticos se clasifican en materiales magnéticamente blandos y magnéticamente duros. En la Figura 2 se ilustran los ciclos de histéresis para el caso de un material magnético blando (Izq.) y duro (Der.). Se nota que para el primero, el ciclo es estrecho con bajos valores de $H_{c}$ (hasta 30 Oe o

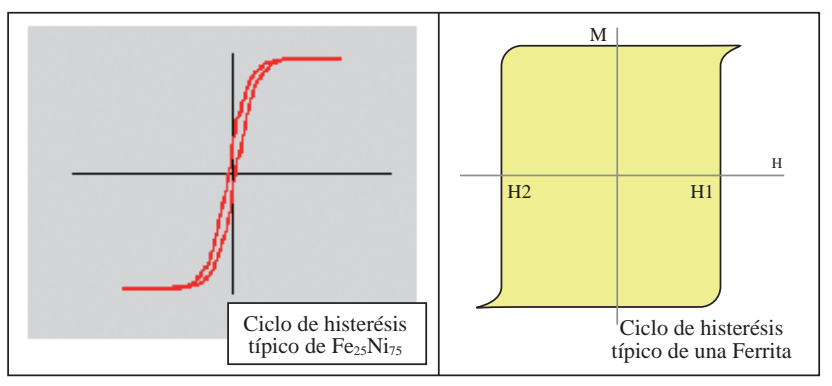

Figura 2. Ciclos de histéresis de materiales blandos (Izq.) y duros (Der.). 
$2400 \mathrm{~A} / \mathrm{m}$ ) y grandes valores de $B_{\mathrm{s}}(>4000$ gauss o $>0.4 \mathrm{~T}$ ), mientras que en el segundo, el ciclo es ancho con grandes valores de $H_{c}\left(>12.6 \mathrm{kOe} 0>10^{6} \mathrm{~A} / \mathrm{m}\right.$ ) y altos valores de $B_{r}$ y $B_{s}(>4000$ gauss o $>0.4 \mathrm{~T}$ ). Materiales magnéticos con valores intermedios de $H_{c}$ son llamados semiduros o semiblandos. Los materiales blandos son ampliamente utilizados en la fabricación de transformadores (Ej. aleaciones $\mathrm{Fe}-\mathrm{Si}$ o $\mathrm{Fe}-\mathrm{Ni}$ ), los duros en imanes permanentes (Ej. $\mathrm{SmCo}_{5}$ y $\mathrm{Nd}_{2} \mathrm{Fe}_{14} \mathrm{~B}$ ), y los semiduros, con ciclos rectangulares, en memorias magnéticas (Ej. aleaciones Nd-Fe).

\section{b. Campo desmagnetizante}

La Figura 2S, http://www.raccefyn.co/index.php/raccefyn/ article/downloadSuppFile/361/1516, (Izq.) ilustra una barra de material ferromagnético, con un eje mucho mayor que los otros dos. La Figura 2S (Der.) ilustra el efecto de aplicar un campo paralelo, $\mathrm{H}_{1}$, y perpendicular, $\mathrm{H}_{2}$, al eje mayor.

Se puede notar que se requiere un campo menor para magnetizar la muestra a lo largo del eje mayor. La apariencia de la curva de magnetización de un material no depende solo de las propiedades magnéticas del material, también depende de la forma de la muestra, lo mismo sucede con su susceptibilidad.

Para entender el porqué de este comportamiento, se utiliza una barra inicialmente no imantada (Figura 3S, http://www.raccefyn.co/index.php/raccefyn/article/download SuppFile/361/1517) a la cual se le aplica un campo externo $H$ de izquierda a derecha, produciendo una magnetización $M$ y una inducción magnética $B$.

Cuando $H$ se retira, el material queda magnetizado en la dirección del campo que fue aplicado, dando lugar al imán o dipolo magnético, pero ahora aparece un campo al interior, entre los polos norte y sur inducidos, contrario al campo que fue aplicado y a la magnetización. Este campo es llamado campo desmagnetizante, $H_{d}$. El campo desmagnetizante es proporcional a la magnetización y ellos se relacionan como

$$
H_{d}=-N_{d} M
$$

donde $N_{d}$ es el factor desmagnetizante, que depende de la geometría del material. Cálculos de $N_{d}$ para diferentes geometrías y direcciones pueden ser encontrados en las referencias [8, 9]. Este campo hay que tenerlo en cuenta en la ecuación (1), la cual en ausencia de $H$ queda de la forma

$$
4 \pi M=B+H_{d} \text { (cgs) o } \mu_{0} M=B+\mu_{0} H_{d} \text { (SI) }
$$

Con el concepto de $H_{d}$ se puede ahora explicar el comportamiento mostrado en la Figura 2S. Cuando el campo se aplica a lo largo del eje mayor de la barra, los polos inducidos están más separados y el campo desmagnetizante es menor que el producido cuando el mismo campo externo se aplica a lo largo del eje menor, porque en este caso los polos estarán más cercanos y el campo desmagnetizante será mayor.

\section{c. Punto de trabajo de un imán permanente}

En la Figura 3 se ilustra el comportamiento de un imán permanente en los ciclos de magnetización y desmagnetización hasta alcanzar el campo coercitivo, $-H_{c}$, pasando por la inducción magnética remanente, $B_{r}$. Cuando el campo $H$ se apaga, la inducción del imán no se queda en $B_{r}$ (y la magnetización en $M_{r}$ ) ya que cuando el campo $H$ se aplicó, el imán se magnetizó y apareció un campo desmagnetizante $-H_{d}$, contrario a $H$. Por lo tanto la curva de desmagnetización va hasta el punto P, donde el campo es $-H_{d}$ y la inducción magnética es $B_{d}$. P es conocido como el punto de trabajo.

El punto P y la línea OC o línea de carga, serán diferentes para diferentes materiales, y también para diferentes geometrías del mismo material. Para un mismo material y diferentes formas se pueden obtener diferentes energías almacenadas en el imán. La pendiente de la línea de carga es dada por

$$
-\frac{4 \pi-N_{d}}{N_{d}}[c g s] o-\frac{\mu_{0}-N_{d}}{N_{d}}[S I]
$$

La Figura 4S, http://www.raccefyn.co/index.php/raccefyn/article/ downloadSuppFile/361/1518, ilustra las curvas hipotéticas de desmagnetización de imanes diferentes 1 y 2, junto con curvas del producto $\mathrm{BH}$ constantes, cuyos valores se ilustran en el eje horizontal superior. Para el imán 1 su curva de desmagnetización podría tocar una de $B H$, si su valor fuese 3 MGOe. Para el imán 2 este corte se da aproximadamente en 2 MGOe. En estos puntos de corte se tendrá para cada imán su valor $(B H)_{\max }$. El diseñador del IP debe variar la geometría del imán con el fin de que el punto de trabajo, $\mathrm{P}$, de lugar a un $(B H)_{\max }$. Las unidades del producto $B H$ están dadas en GOe o MGOe [cgs] y en $\mathrm{kJ} / \mathrm{m}^{3}$ [SI] y su relación es MGOe $/ \mathrm{kJ} / \mathrm{m}^{3}=7.96$. El valor de $B H$ corresponde al área

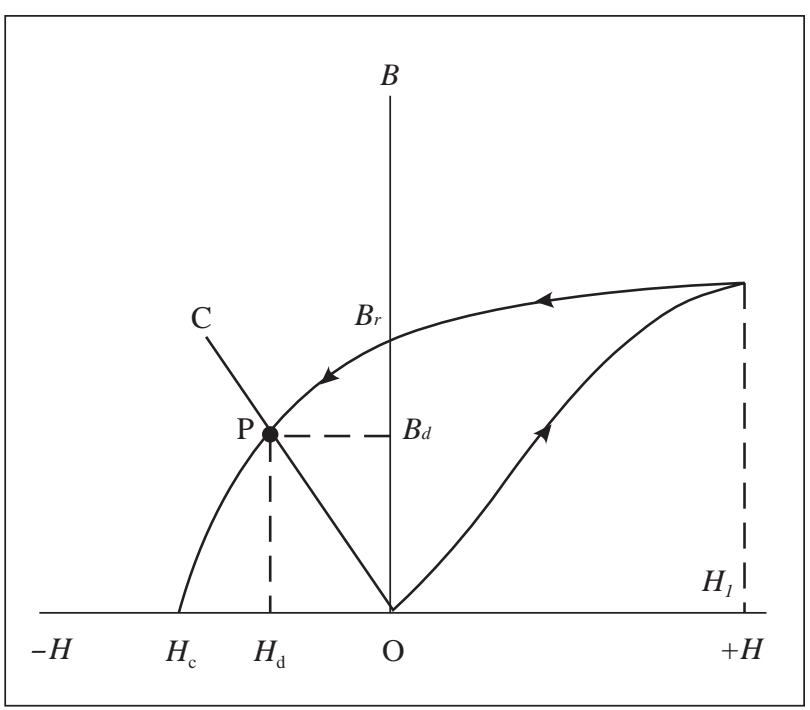

Figura 3. Curva de magnetización inicial y de desmagnetización de un imán permanente. $\mathrm{B}_{\mathrm{r}}$ es la inducción remanente y $\mathrm{P}$ es el punto de trabajo. 
del rectángulo de base $H_{d}$ y altura $B_{d}$ de la Figura 3, o al rectángulo de base $H_{m}$ y altura $B_{m}$ de la Figura 1S. Algunos operadores trabajan con el valor de la pendiente de la línea de carga o $B_{d} / H_{d}=B_{m} / H_{m}$, que es la permeabilidad magnética del material. Estas pendientes y sus valores se ilustran en el eje vertical izquierdo y el horizontal superior. Encontrar sus valores no depende del conocimiento de los valores de $N_{d}$.

\section{d. Anisotropía cristalina}

En la Figura 5S, http://www.raccefyn.co/index.php/raccefyn/ article/downloadSuppFile/361/1519, se muestran las curvas de magnetización inicial para un cristal simple de Fe (Izq.) y otro de Ni (Der.), para diferentes direcciones dentro del cristal. Se puede notar que para el Fe se alcanza la saturación aplicando menor campo a lo largo de la dirección $<100>$ y con mayor campo a lo largo de la $<111>$. Por esta razón la dirección $<100>$ es llamada dirección fácil y la $<111>$ es la dirección difícil o dura. Para el Ni el eje fácil es el $<111>$ y el duro el $<100>$. Las seis direcciones equivalentes [100], [010], [001], [100], [010] y [001] , se representan por la notación $<100>$. Esta propiedad de los materiales es conocida como anisotropía magnética cristalina.

En la Figura 6S, http://www.raccefyn.co/index.php/ raccefyn/article/downloadSuppFile/361/1520, se muestra una vista esquemática del plano $\{001\}$ del Fe, inicialmente desmagnetizado, donde los dominios se orientan en las dos direcciones fáciles que se encuentran en este plano, o sea las direcciones [100] y [010] (ver Fig. 6Sa), con un valor de magnetización $M_{s}$. Cuando se aplica un campo $H$ en la dirección [110], que forma ángulos de $45^{\circ}$ con las direcciones [100] y [010], los dominios con ángulos de $45^{\circ}$ con $H$ crecen y los de $135^{\circ}$ decrecen (ver Fig. 6Sb). Si el campo aumenta, los dominios de menor ángulo con el campo aumentan de tamaño y los de mayor ángulo disminuyen. Esta tendencia continúa hasta cuando el campo es tal que solamente se tendrán dominios formando ángulos de $45^{\circ}$ con el campo (ver Fig. 6Sc). Si el campo $H$ sigue aumentando, se llegará a la situación en la cual todos los dominios apuntan a lo largo del campo y se tendrá un solo dominio o monodominio (ver Fig. 6Sd). En esta situación el Fe alcanzará la magnetización de saturación.

La mayor o menor dificultad para magnetizar un material en una determinada dirección implica una mayor o menor energía para conseguirlo. Akulov (1936) en 1936 postuló una expresión para la energía de anisotropía, por unidad de volumen, para cristales cúbicos, cuando la magnetización de saturación $M_{s}$ forma ángulos $\alpha, \beta$ y $\gamma$ con los ejes cristalinos, de la forma

$$
E=K_{0}+K_{1}\left(\alpha_{1}^{2} \alpha_{2}^{2}+\alpha_{2}^{2} \alpha_{3}^{2}+\alpha_{3}^{2} \alpha_{1}^{2}\right)+K_{2}\left(\alpha_{1}^{2} \propto_{2}^{2} \alpha_{3}^{2}\right)+\cdots
$$

donde $\alpha_{1}=\cos \alpha, \alpha_{2}=\cos \beta$ y $\alpha_{3}=\cos \gamma$, son los cosenos directores. $K_{0}, K_{1}, K_{2}, \ldots$ son constantes que dependen del material y de la temperatura. Están expresadas en erg/ $/ \mathrm{cm}^{3}$ o $\mathrm{J} / \mathrm{m}^{3}$. En la Tabla 1, se ilustran los valores de la energía de anisotropía para diferentes direcciones de la magnetización en un cristal cúbico. Si $K_{1}$ y $K_{2}$ son positivos (caso del Fe) se nota que la menor energía se obtiene cuando $M_{\mathrm{s}}$ es paralelo a la dirección fácil [100] y mayor en la dirección dura [111]. Para el Ni $K_{1}$ y $K_{2}$ son negativos y la menor energía se obtiene cuando $M_{s}$ es paralela a la dirección [111], que será la dirección fácil. La mayor energía se obtiene en la dirección [100] y esta será la dirección dura del Ni.

Medidas experimentales muestran que para el Fe, $K_{1}=$ $4.8 \times 10^{5} \mathrm{erg} / \mathrm{cm}^{3}=480 \mathrm{~kJ} / \mathrm{m}^{3}$ y $K_{2}=0.5 \times 10^{5} \mathrm{erg} / \mathrm{cm}^{3}=50$ $\mathrm{kJ} / \mathrm{m}^{3}$. Para el Ni se reportan $K_{1}=-0.5 \times 10^{5} \mathrm{erg} / \mathrm{cm}^{3}=-50 \mathrm{~kJ} /$ $\mathrm{m}^{3}$ y $K_{2}=-0.2 \times 10^{5} \mathrm{erg} / \mathrm{cm}^{3}=-20 \mathrm{~kJ} / \mathrm{m}^{3}$.

Para el caso de cristales hexagonales las curvas de magnetización en diferentes direcciones son mostradas en la Figura 7S, http://www.raccefyn.co/index.php/raccefyn/ article/downloadSuppFile/361/1521.

Se puede notar que a lo largo del eje mayor, o eje $c$, con poco campo se consigue la magnetización de saturación $M_{\mathrm{s}}$ (eje de fácil magnetización), mientras que en un eje basal se necesita mucho más campo para conseguir la saturación (eje de difícil magnetización o eje duro).

Para este tipo de cristales la densidad de energía magnetocristalina propuesta es dada por

$$
E=K_{0}+K_{1} \operatorname{sen}^{2} \theta+K_{2} \operatorname{sen}^{4} \theta+\cdots
$$

Donde $\theta$ es el ángulo que la magnetización $M_{s}$ forma con el eje fácil. Este tipo de cristal se conoce como uniaxial (ver Figura 8S, http://www.raccefyn.co/index.php/raccefyn/ article/downloadSuppFile/361/1522), mientras que el del Fe es triaxial.

Los valores experimentales de las constantes de anisotropía encontrados para el caso del Co son $K_{1}=4.8 \times 10^{6} \mathrm{erg} / \mathrm{cm}^{3}$ $=480 \mathrm{~kJ} / \mathrm{m}^{3}$ y $K_{2}=1.5 \times 10^{6} \mathrm{erg} / \mathrm{cm}^{3}=150 \mathrm{~kJ} / \mathrm{m}^{3}$.

\section{e. Anisotropía de forma}

La Figura 9S, http://www.raccefyn.co/index.php/raccefyn/ article/downloadSuppFile/361/1523, muestra un material magnético en forma de elipsoide prolata, con eje mayor $c$ y menor $a$, se sabe que es más fácil magnetizarlo a lo largo del eje $c$ (eje fácil), que a lo largo del eje $a$ (eje duro). Esto se debe a que el campo desmagnetizante es menor a lo largo de $c$ que a lo largo de $a$. Si la magnetización $M$ forma un

Tabla 1. Valores de energía de anisotropía para un cristal cúbico en diferentes direcciones cristalográficas.

\begin{tabular}{cccccccc}
\hline$[\mathbf{k l m}]$ & $\boldsymbol{\alpha}$ & $\boldsymbol{\beta}$ & $\boldsymbol{\gamma}$ & $\boldsymbol{\alpha}_{1}$ & $\boldsymbol{\alpha}_{2}$ & $\boldsymbol{\alpha}_{3}$ & $\boldsymbol{E}$ \\
\hline$[100]$ & 0 & $90^{\circ}$ & $90^{\circ}$ & 1 & 0 & 0 & $K_{0}$ \\
{$[110]$} & $45^{\circ}$ & $45^{\circ}$ & $90^{\circ}$ & $\sqrt{ } 2 / 2$ & $\sqrt{ } 2 / 2$ & 0 & $K_{0}+K_{1} / 4$ \\
{$[111]$} & $54.74^{\circ}$ & $54.74^{\circ}$ & $54.74^{\circ}$ & $1 / \sqrt{ } 3$ & $1 / \sqrt{ } 3$ & $1 / \sqrt{ } 3$ & $K_{0}+K_{1} / 3+K_{2} / 27$ \\
\hline
\end{tabular}


ángulo $\theta$ con el eje mayor $c$, se puede mostrar (B. D. Cullity \& C. D. Graham) que la energía potencial por unidad de volumen es dada por

$$
E=\frac{1}{2} M^{2} N_{C}+\frac{1}{2}\left(N_{a}-N_{c}\right) M^{2} \operatorname{sen}^{2} \theta
$$

Donde $N_{a}$ y $N_{c}$ son las constantes desmagnetizantes a lo largo de $a$ y $c$, respectivamente. La forma de esta expresión es similar a la de la energía de anisotropía propuesta para sistemas uniaxiales, con $K_{0}=M^{2} N_{c} / 2$ siendo la energía a lo largo del eje fácil y el segundo término, $K_{1}=\left(N_{a}-N_{c}\right)$ $M^{2} / 2$, es la energía que hay que darle al sistema para que $M$ cambie su orientación desde $c$, hasta formar un ángulo $\theta$ con este eje.

Existen otras formas de anisotropía magnética que pueden ser encontradas, tales como la anisotropía por tensión, inducida, de intercambio, etc., pero las principales son las ya mostradas. Para imanes nanoestructurados la tercera también es importante

\section{Materiales para imanes permanentes (B. D. Cullity \& C. D. Graham y O'Hanley)}

\section{a. Aceros magnéticos}

El primer material utilizado como imán permanente fue la piedra imán o la conocida magnetita, originalmente encontrada en Magnesia, Grecia. Presenta $H_{c}$ de algunas decenas de Oe y $B_{s}$ entre 0.3 y 0.4 T. Fue usado por los chinos en agujas de brújula, pero hoy en día no tiene aplicaciones como material en bulto, pero si en forma de nanopartículas. Posteriormente fueron utilizados los imanes de aceros de alto carbón, endurecidos por temple, que se usaron durante muchos siglos como agujas de las brújulas. La presencia del $\mathrm{C}$ en el Fe lo endurece mecánica y magnéticamente, ya que genera cementita, $\mathrm{Fe}_{3} \mathrm{C}$, que impide el transporte de dislocaciones y de paredes de dominio. El campo coercitivo es de 50 Oe (4 kA/m). Se usó hasta mediados del siglo 19.

A finales del siglo 19 los aceros con $\mathrm{W}$ fueron usados para herramientas de corte y aplicaciones estructurales, y en 1885 un acero con $5 \% \mathrm{~W}$ se usó como imán. Este fue reemplazado, durante la $1^{\text {a }}$ Guerra Mundial por un acero al Cr más barato, presentando $H_{c}=100$ Oe $(8 \mathrm{kA} / \mathrm{m})$. En 1917 Honda y Takagi mostraron que un acero con 30-40 \% Co, más $\mathrm{W}$ y Cr, mostraba $H_{c}=230$ Oe, $B_{s}=2.4 \mathrm{~T}$ y $(H B)_{\max }=1$ MGOe. Este continúa siendo el mejor imán hecho de acero en bulto. En 1961 Luborky preparó un imán mediante la sinterización de nanopartículas monodominio alargadas de FeCo, con tamaños entre 10 y $15 \mathrm{~nm}$. Obtuvo coercitividades del orden $2 \mathrm{kOe}(140 \mathrm{kA} / \mathrm{m})$.

\section{b. Alnico}

Es el nombre genérico de una familia de aleaciones con base a Fe, Co, Ni más pequeñas cantidades de $\mathrm{Al}, \mathrm{Cu}$ y otros elementos. Fe es el elemento mayoritario. En cierta época fueron los imanes más utilizados, pero hoy en día son las ferritas duras y los imanes de tierras raras los que dominan el mercado.
Mishima en 1932 encontró que una aleación con 58\% Fe, $30 \% \mathrm{Ni}$ y $12 \% \mathrm{Al}$ mostraba un campo coercitivo de 400 Oe $(5.7 \mathrm{~A} / \mathrm{m})$. Posteriormente se mostró que agregar Co y $\mathrm{Cu}$ mejoraba sus propiedades. La numeración del alnico depende de la composición.

Los alnico son duros y frágiles y de granos gruesos cuando se fabrican por colada o sinterización. Si un tratamiento térmico se le hace con las siguientes tres etapas, sus propiedades mejoran:

i) Tratamiento a $1500{ }^{\circ} \mathrm{C}$ hasta su homogenización.

ii) Enfriamiento a una rata de $1{ }^{\circ} \mathrm{C} / \mathrm{s}$ hasta $\sim 500{ }^{\circ} \mathrm{C}$.

iii) Recalentada durante varias horas a $600{ }^{\circ} \mathrm{C}$ (Envejecido).

Algunos alnico son enfriados en presencia de campo, aumentando así la coercitividad del material en la dirección del campo. Este tratamiento hace que se precipite una fase ferromagnética, bcc rica en $\mathrm{Fe}(\alpha)$, en una matriz débilmente magnéticamente, bcc $\mathrm{NiAl}\left(\alpha^{\prime}\right)$, (descomposición espinoidal, ver Figura 10S, http://www.raccefyn.co/index.php/raccefyn/ article/downloadSuppFile/361/1524). Ellas se precipitan como barras alternadas de $300 \AA$ A de diámetro. Si se aplica un campo en la etapa de precipitación ellas se orientan a lo largo de la dirección [100] y no en las tres direcciones $<100>$.

La Figura 11S, http://www.raccefyn.co/index.php/raccefyn/ article/downloadSuppFile/361/1525, muestra una micrografía electrónica del alnico 5 a la cual se le aplicó un campo en la dirección horizontal. Se observa en esta figura que los granos son alargados en la dirección del campo y se tienen granos alternados de la fase ferromagnética y la débilmente magnética. La morfología de los granos da lugar a una anisotropía de forma, responsable del carácter duro de estos imanes.

Una manera de mejorar el carácter duro de estas aleaciones se consigue produciendo una textura cristalográfica con los granos a lo largo de [100]. Esto se obtiene por solidificación direccional con los granos creciendo en la dirección del flujo de calor (ver Figura 12S, http://www.raccefyn.co/index.php/ raccefyn/article/downloadSuppFile/361/1526). Si además se aplica campo en esta dirección, se puede mejorar aún más el carácter duro de la aleación.

La Figura 13S, http://www.raccefyn.co/index.php/raccefyn/ article/downloadSuppFile/361/1527, ilustra curvas de desmagnetización de algunos alnicos. Se puede notar que existe una mejora en el producto $(B H)_{\max }$ del alnico 5 cuando se incrementa su anisotropía de forma, pasando de 60 a $80 \mathrm{~kJ} / \mathrm{m}^{3}$.

La investigación de los alnicos básicamente terminó cuando fueron descubiertos los imanes de tierras raras.

\section{c. Ferritas de bario y estroncio}

Las ferritas duras constituyen el mayor mercado de los IPs y se usan en aplicaciones donde la energía por unidad de peso y costo sea importante. 
Las ferritas de $\mathrm{Ba}, \mathrm{BaO} .6\left[\mathrm{Fe}_{2} \mathrm{O}_{3}\right]$, de $\mathrm{Sr}$, $\mathrm{SrO} .6\left[\mathrm{Fe}_{2} \mathrm{O}_{3}\right]$ y de $\mathrm{Pb}, \mathrm{PbO} .6\left[\mathrm{Fe}_{2} \mathrm{O}_{3}\right.$ ], presentan la estructura magnetoplumbita. Se denotan como BaM, SrM y PbM. Estas ferritas hexagonales o hexaferritas presentan una alta anisotropía cristalina a lo largo del eje fácil $c$, del orden de $3.3 \times 10^{6} \mathrm{erg} / \mathrm{cm}^{3}$ o 330 $\mathrm{kJ} / \mathrm{m}^{3}$. La magnetización de saturación es baja, pero su alta anisotropía da lugar a altos valores de $(\mathrm{BH})_{\max }$, del orden de $\mathrm{kJ} / \mathrm{m}^{3}$.

La primera ferrita de Ba comercial se produjo en 1952 por la Philips. Normalmente se produce por mezcla de $\mathrm{BaCO}_{3}$ (o $\mathrm{SrCO}_{3}$ o $\mathrm{PbCO}_{3}$ ) y $\mathrm{Fe}_{2} \mathrm{O}_{3}$, seguida de homogenización a 1200 ${ }^{\circ} \mathrm{C}$ (calcinación), hasta obtener la ferrita. Este material se lleva a un molino de bolas para obtener tamaños de partícula del orden de $\mu \mathrm{m}$, estas partículas presentan formas de placas hexagonales. También se obtienen por solución de $\mathrm{Fe}_{2} \mathrm{O}_{3}$ y $\mathrm{BaO}$ dando lugar a precipitación de placas hexagonales, tal como se observa en la Figura 14S, http://www.raccefyn.co/ index.php/raccefyn/article/downloadSuppFile/361/1528.

La anisotropía de este material es principalmente cristalina, con eje fácil perpendicular al plano hexagonal. A lo largo de este eje se obtiene $H_{c} \sim 17 \mathrm{kOe}$ (cgs) $01.7 \mathrm{~T}$ (SI) para una partícula. Sin embargo la morfología que se obtiene en su fabricación, da lugar a una anisotropía de forma paralela al plano hexagonal, que es perpendicular al eje fácil. Esto reduce el valor de $H_{c}$ por partícula hasta $12 \mathrm{kOe}$. Este material es luego prensado en medio húmedo aplicando un campo a lo largo del eje de presión. La microestructura que se obtiene es mostrada en la figura $15 \mathrm{~S}$, http://www.raccefyn. co/index.php/raccefyn/article/downloadSuppFile/361/1529. En la Figura 15S (Izq.) se tiene una vista paralela al eje fácil y en la 15S (Der.) perpendicular al eje fácil.

Para mejorar sus características se refina el tamaño de partícula. El valor máximo obtenido para $(B H)_{\max }$ es del orden de $3.5 \mathrm{MGOe}$ o $28 \mathrm{~kJ} / \mathrm{m}^{3}$, y no ha sido mejorado en muchos años.

\section{d. Imanes de tierras raras}

Los elementos de tierras raras (TR, número atómico entre 59 y 70) son ferromagnéticos con temperaturas de Curie $\left(\mathrm{T}_{\mathrm{c}}\right)$ abajo de la temperatura ambiente $\left(\mathrm{T}_{\mathrm{a}}\right)$ y con gran anisotropía magnética. Cuando se alean con elementos de transición (ET) como Fe, Co o Ni dan compuestos con alta anisotropía, debido a la tierra rara, ya que esta no se modifica apreciablemente con otros elementos (anisotropía de átomo simple).

El primer IP de este tipo fue el $\mathrm{YCo}_{5}$ descubierto por Strnat and Goffer en 1966, que presentó una constante de anisotropía de $5.5 \times 10^{7} \mathrm{erg} / \mathrm{cm}^{3}$ o $5.5 \times 10^{3} \mathrm{~kJ} / \mathrm{m}^{3}$, por mucho la mayor encontrada en esa época. A partir de allí mucho trabajo se ha realizado en sistemas de TR/ET buscando mejores propiedades (una mayor anisotropía cristalina uniaxial, alta $\mathrm{T}_{c}$, alto valor de $\mathrm{B}_{s}$, bajo costo, buenas propiedades mecánicas y baja densidad.
Tres familias de IPs tipo TR/ET han sido investigadas: $\mathrm{SmCo}_{5}$ (mayor $\mathrm{K}$ ), $\mathrm{Sm}_{2} \mathrm{Co}_{17}$ (mayores flujo magnético y $\mathrm{T}_{\mathrm{c}}$ ) y $\mathrm{Nd}_{2} \mathrm{Fe}_{14} \mathrm{~B}$ (mayor $(\mathrm{BH})_{\max }$ ), todas ellas con producto $(B H)_{\max }$ muy superior a los mejores alnico y hexaferritas.

\section{d.1 Imanes de Co/TR}

Los más importantes IPs con base al sistema SmCo contienen grandes cantidades de Co. En la figura 16S, http://www.raccefyn.co/index.php/raccefyn/article/ downloadSuppFile/361/1530, se muestra el diagrama de fases de equilibrio del sistema SmCo en la región rica en Co. Varias fases estables se pueden encontrar, por eso, dependiendo de la composición y de los tratamientos térmicos y mecánicos, se pueden obtener IPs con una sola fase o con varias de las fases.

Una de las fases que aparece en el diagrama es la del $\mathrm{SmCo}_{5}$, o fase1-5, que tiene una estructura hexagonal con eje fácil a lo largo de $c$, y una constante de anisotropía de $7.7 \times 10^{7} \mathrm{erg} / \mathrm{cm}^{3}=7.7 \times 10^{3} \mathrm{~kJ} / \mathrm{m}^{3}$. Se obtiene por fusión y colada, luego trituración y molienda hasta obtener tamaños de partícula del orden de $10 \mu \mathrm{m}$, cada partícula siendo un monocristal de $\mathrm{SmCo}_{5}$. El polvo es alineado en un campo para alinear los cristales con $c$ paralelo al campo y luego prensado en seco. Finalmente es sinterizado a más de $1000{ }^{\circ} \mathrm{C}$ para producir el IP final.

Para obtener un material con mayor densidad se agrega al polvo antes de sinterizar un compuesto rico en Sm, de tal manera que este se licúe durante la sinterización, llenando los espacios entre partículas. Este proceso es conocido como sinterización en fase líquida (SFL). El tamaño de grano obtenido es el mismo de las partículas antes del proceso de SFL, y como este tamaño es del orden de 10 veces el tamaño de dominio, los granos serán multidominio. Para este tipo de IP se obtiene una curva de magnetización y desmagnetización como la de la Figura 17S, http://www.raccefyn.co/index. php/raccefyn/article/downloadSuppFile/361/1531.

Se puede notar que el campo para saturar es mucho menor que $H_{c}$, contrario al de la mayoría de los IPs, donde el campo necesario para saturar es mayor que $H_{c}$. Esto se debe a que los granos son multidominio y sus paredes de dominio se pueden mover fácilmente en la dirección del campo hasta ser expulsadas y obtener un grano monodominio. Para generar un dominio en dirección contraria se necesita un gran campo.

Los imanes tipo $\mathrm{SmCo}_{5}$ fueron los primeros en llegar a un producto de energía de $20 \mathrm{MGOe} \sim 160 \mathrm{~kJ} / \mathrm{m}^{3}$.

Otra de las fases que aparece en el diagrama SmCo es la $\mathrm{Sm}_{2} \mathrm{Co}_{17}$, o fase 2-17, cuya estructura es romboédrica. Se trabajan también compuestos donde el Co es sustituido por $\mathrm{Fe}, \mathrm{Cu}$ y $\mathrm{Zr}$, lo cual los hace más baratos. Se producen de manera similar a la tipo $1-5$, pero con tratamientos adicionales que hacen que la microestructura sea muy fina con camadas de fase 1-5 separando las 2-17. Esta estructura 
fina produce un anclaje de las paredes de dominio haciendo que $H_{c}$ sea alto. La anisotropía magneto cristalina de las fases 2-17 $\left(3-4 \times 10^{3} \mathrm{~kJ} / \mathrm{m}^{3}\right)$ es menor que la de la fase $1-5$ (11-20x $\left.10^{3} \mathrm{~kJ} / \mathrm{m}^{3}\right)$, pero los valores de $M_{s}$ y $T_{c}$ son mayores.

La estructura romboédrica de los compuestos 2-17 permite que se agregue más Fe que en las 1-5. Coercitividades del orden de $800 \mathrm{kA} / \mathrm{m}$ (10 kOe) y productos de energía de $340 \mathrm{~kJ} / \mathrm{m}^{3}$ (30MGOe) se alcanzan para el compuesto $\mathrm{Sm}_{2}(\mathrm{Co}, \mathrm{Fe})_{17}$.

\section{d.2 Imanes intermetálicos con base a $\mathrm{Nd}_{2} \mathrm{Fe}_{14} \mathrm{~B}$}

Como el Fe y el Nd son más baratos que el Co y el Sm, respectivamente, esfuerzos independientes, para producir mejores y más baratos imanes, llevaron a Das \& Koon (1981), a Croat (1981) y a Hadjipanayis, Hazelton \& Lawless (1983-4), a producir, por diferentes métodos, IPs tipo $\mathrm{Nd}_{2} \mathrm{Fe}_{14} \mathrm{~B}$ o tipo 2-14-1. Comercialmente se produjeron simultáneamente imanes tipo $\mathrm{Nd}_{2} \mathrm{Fe}_{14} \mathrm{~B}$ por Sagawa,

Fujimura, Togawa \& Matsura (1984) (Sumimoto Special Metals Co.) y Croat, Herbst, Lee \& Pinlterton (1984) (General Motors Corp.). Estos imanes presentan una estructura tetraédrica con una gran anisotropía magnetocristalina $\left(5 \times 10^{3} \mathrm{~kJ} / \mathrm{m}^{3}\right)$. El Fe presenta 6 sitios diferentes en esta estructura, el Nd 2 y el B 1. Si se coloca Co en los sitios del Fe se aumenta $T_{c}$ y la estabilidad térmica; y si se reemplaza la TR liviana Nd por la pesada Dy aumenta la coercitividad. Estas combinaciones dan lugar a productos de energía de hasta 50 MGOe $\left(398 \mathrm{~kJ} / \mathrm{m}^{3}\right.$ ) y $B_{s}$ del orden de $1.6 \mathrm{~T}$, debido al gran acople ferromagnético entre el Fe y el Nd. Sus principales desventajas son su baja $T_{c}$, del orden de $300{ }^{\circ} \mathrm{C}$ y su baja resistencia a la corrosión.

Estas aleaciones pueden producirse por diferentes métodos tales como fundición y tratamientos térmicos para inducir la nanoestructura deseada; enfriamiento rápido para producir cintas amorfas que luego son tratadas térmicamente para inducir la cristalización de la(s) fase(s) deseada(s), para luego ser pulverizadas y sinterizadas en la forma final del IP; y por aleamiento mecánico. Diferentes tipos de piezas que se pueden producir y que están disponibles en el mercado se ilustran en la Figura 18S, http://www.raccefyn. co/index.php/raccefyn/article/downloadSuppFile/361/1532.

En la producción de algunos IP tipo $\mathrm{Nd}_{2} \mathrm{Fe}_{14} \mathrm{~B}$ actualmente se coloca un exceso de Fe y B y la microestructura que se obtiene, después de los tratamientos térmicos, es compuesta de granos nanométricos de la fase dura 2-14-1 separados por granos nanométricos de fase blanda tipo $\alpha$-Fe o FeB. Cuando el exceso es de $\mathrm{Nd}$ y/o B la nueva fase es no magnética rica en $\mathrm{B}$ o Nd. Para el primer caso la fase dura da la alta anisotropía o alto $H_{c}$ y la blanda una gran $M_{r}$.

En la Figura 4, Hadjipanayis (2008), se ilustra cómo ha evolucionado el valor $(\mathrm{BH})_{\max }$ (arriba) y la nanoestructura (abajo) de los IPs con base a Nd-Fe-B. Se supone que el imán está en el punto de trabajo con el $H_{d}$ hacia abajo y $M_{s}\left(B_{s}\right)$ hacia arriba. En el primer esquema de la izquierda (abajo) se muestra un IP constituido de granos de fase dura $\mathrm{Nd}_{2} \mathrm{Fe}_{14} \mathrm{~B}$ (azul) con orientación cristalina aleatoria (isotrópica) separados por una fase no magnética (rica en Nd y/o B). En este caso $(B H)_{\max }$ es del orden de 12 MGOe. En la segunda no existe exceso de elementos no magnéticos y no aparece la fase paramagnética rodeando los granos de la fase dura y estos presentan orientación cristalina al azar pero ahora existe un acople de intercambio ferromagnético entre ellos. Este acople aumenta la magnetización y por ende el producto $(B H)_{\max }$ que ahora es del orden de 20 MGOe. La tercera nanoestructura se debe al exceso de Fe o/y B en la composición inicial, dando lugar al aparecimiento de granos blandos de $\alpha-\mathrm{Fe}$ y/o $\mathrm{Fe}_{2} \mathrm{~B}$ (amarillo). Se obtiene entonces un nanocompuesto. Existe acople ferromagnético entre granos

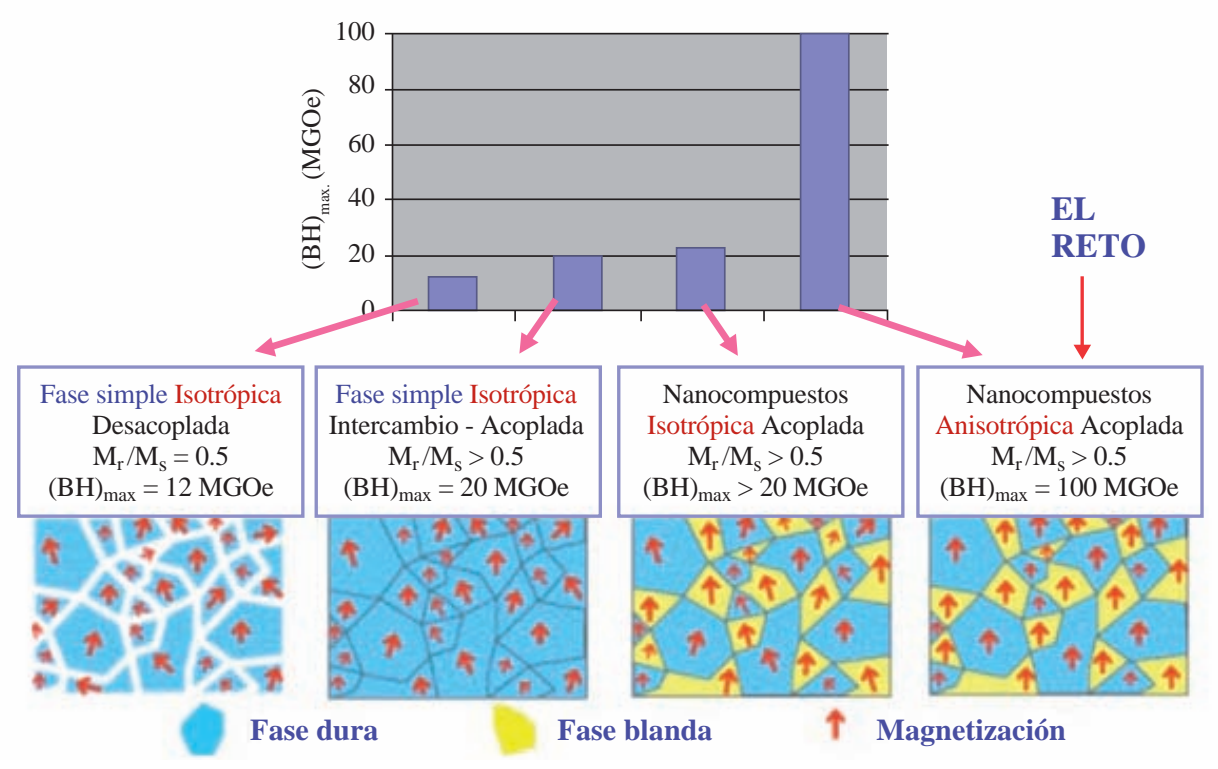

Figura 4. Desarrollo histórico de los IPs nanoestructurados de Nd-Fe-B (Hadjipanayis (2008)). 
duros y blandos dando lugar a mayor magnetización, además los granos blandos dan lugar a mayor magnetización que los granos duros. De esta manera se alcanza un $(\mathrm{BH})_{\max }$ mayor a 20 MGOe. Finalmente lo que se busca es lo mostrado en el esquema de la derecha donde los granos blandos y duros estén con sus ejes fáciles en la misma dirección (anisotropía) y acoplados ferromagnéticamente para obtener valores de $(B H)_{\text {max }}$ del orden de 100 MGOe.

En la Figura 19S, http://www.raccefyn.co/index.php/ raccefyn/article/downloadSuppFile/361/1533, se muestran las características magnéticas de los imanes nanocompuestos tipo Nd-Fe-B.

El método como se han alcanzado altos valores de $(B H)_{\max }$, con el cual se espera obtener un IP nanocompuesto isotrópico con mayores valores de $(B H)_{\max }$, es utilizando el método de deformación plástica en caliente (DPC o Die-upseting), el cual se ilustra en la Figura 5 (Izq.).

En la Figura 5 (Der.) se comparan las curvas de desmagnetización obtenidas para imanes de Nd-Fe-B en bulto. Estos imanes se produjeron por melt-spun, cristalización por tratamiento térmico, pulverización y luego sinterización con epoxi (abajo), compresión isotrópica en caliente (medio), y por DPC caliente. Se puede notar que el método DPC da lugar a una mejora del orden de un $50 \%$ en las propiedades magnéticas.

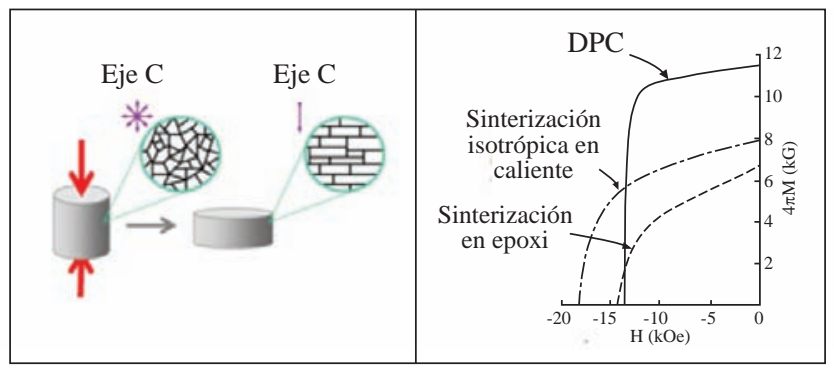

Figura 5. Ilustración del proceso de deformación plástica en caliente (Izq.) (Hadjipanayis (2008)) y efecto de los proceso de sinterización en la curva de desmagnetización de imanes de NdFe-B (Der.) (Herbst (1991)).
En la Tabla 2 se comparan propiedades magnéticas de varios IPs.

En la Figura 6 (Izq.) se ilustra cómo ha evolucionado en los últimos 100 años el valor alcanzado de $(B H)_{\max }$. Se puede notar que en este periodo el valor de $(\mathrm{BH})_{\max }$ ha aumentado en aproximadamente 100 veces. En la Figura 6 (Der.) se compara la manera como el volumen, para producir un campo de $1000 \mathrm{G}$ a $5 \mathrm{~mm}$ de la cara de un polo, ha disminuido en aproximadamente 65 veces, desde el alnico 5-7 al NdFeB.

\section{e. Otros IPs \\ e.1 Imanes CoPt}

Este material presenta valores $B_{s}$ entre 0.2-0.4 T, $H_{c}$ $\sim 340 \mathrm{kA} / \mathrm{m}$ (4.3 kOe) y $T_{c}=550{ }^{\circ} \mathrm{C}$. Presenta una red fcc ferromagnética arriba de $820{ }^{\circ} \mathrm{C}$ y abajo de esta $\mathrm{T}$, para contenidos de Co entre 35 y 65\%, presenta una estructura fcc ordenada con átomos de Pt en la base y la tapa, y átomos de Co en el plano central. Este ordenamiento resulta en una distorsión tetragonal que da lugar a $K$ uniaxial de $5 \times 10^{6} \mathrm{~J} /$ $\mathrm{m}^{3}$ y $H_{c}$ de $6 \mathrm{kOe}$ en la dirección [001]. Este valor de Hc no es de la estructura ordenada y si de una mezcla fina de las estructuras ordenada y desordenada. Se cree que la dureza magnética viene del anclaje de las paredes de dominio en las fronteras de grano, esto implica que los granos son monodominios. Este sistema presenta un $(B H)_{\max }$ entre 70$95 \mathrm{~kJ} / \mathrm{m}^{3}$ (8.5-12 MGOe) y presenta una resistencia a la corrosión mayor que cualquier otro IP metálico. Él juega un rol importante en las películas delgadas de los medios de grabación usados en discos duros.

Los sistemas FePt y FePd también presentan la fase ordenada anisotrópica. Las propiedades magnéticas se mejoran por envejecimiento en campo o por deformación plástica a lo largo del eje [001].

\section{e.2 Imanes MnAl}

La Figura 20S, http://www.raccefyn.co/index.php/raccefyn/ article/downloadSuppFile/361/1534, (Izq.) ilustra el diagrama de fases de equilibrio del sistema MnAl. En este diagrama de fase aparece, en la región cercana a la equiatómica, entre

Tabla 2. Propiedades magnéticas de algunos IPs (Cullity \& Graham (2009)).

\begin{tabular}{|c|c|c|c|c|c|c|}
\hline \multirow[t]{2}{*}{ Imán } & \multirow[t]{2}{*}{$\mu_{0} M_{\mathrm{s}}(\mathrm{T})$} & \multirow[t]{2}{*}{$T_{c}\left({ }^{\circ} \mathrm{C}\right)$} & \multirow[t]{2}{*}{$K\left(\mathrm{MJ} / \mathrm{m}^{3}\right)$} & \multirow{2}{*}{$\begin{array}{c}H_{c}(\mathrm{MA} / \mathbf{m}) \\
\text { Isotrópico }\end{array}$} & \multirow{2}{*}{$\begin{array}{l}H_{c}(\mathrm{MA} / \mathrm{m}) \\
\text { Anisotrópico }\end{array}$} & \multirow[t]{2}{*}{$(B H)_{\max }(\mathrm{MGOe})$} \\
\hline & & & & & & \\
\hline Ferrita & 0.47 & 723-733 & 0.34 & & 0.36 & 4 \\
\hline Alnico 5 & 1.25 & 750 & 0.38 & --- & 0.07 & 5.5 \\
\hline Alnico 9 & 1.12 & 750 & 0.41 & --- & 0.14 & 10.5 \\
\hline $\mathrm{SmCo}_{5}$ & 1.0 & $685-700$ & 10 & $0.8-1$ & 2.9 & $18-24$ \\
\hline $\mathrm{Sm}_{2} \mathrm{Co}_{17}$ & $1.2-1.4$ & $810-970$ & 3.3 & $1-1.3$ & 2.4 & $24-30$ \\
\hline $\mathrm{Nd}_{2} \mathrm{Fe}_{14} \mathrm{~B}$ & 1.6 & 312 & 5 & --- & $1.2-1.6$ & $34-45$ \\
\hline
\end{tabular}




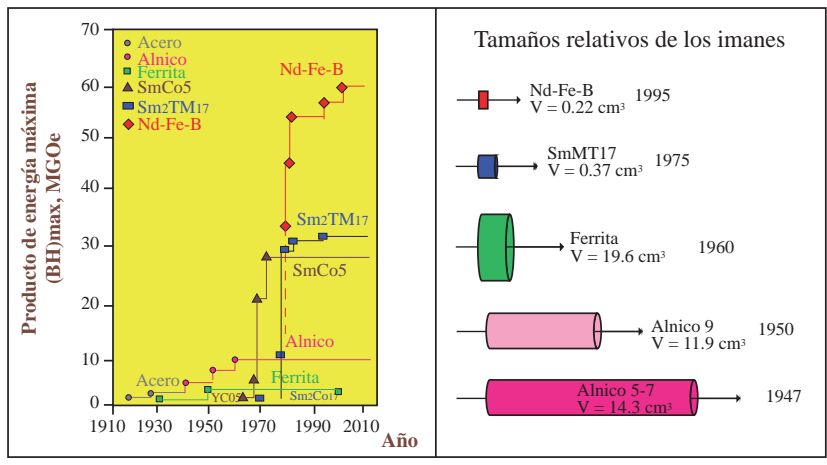

Figura 6. Evolución temporal del valor de $(B H)_{\max }$ (Izq.), y evolución temporal del volumen del imán para producir un campo de $1000 \mathrm{G}$ a $5 \mathrm{~mm}$ de la superficie de un polo (Jiménez-Villacorta \& Lewis (2014)).

52 y $57 \% \mathrm{Mn}$, la fase metastable $\tau$. Esta fase presenta una estructura tetragonal centrada en las caras fct ordenada, Figura 20S (Der.) con átomos de Mn en posiciones (000) y $\left(\begin{array}{lll}1 / 2 & 1 / 2 & 0\end{array}\right)$ y átomos de $\mathrm{Al}$ en posiciones $\left(\begin{array}{lll}1 / 2 & 1 / 2\end{array}\right)$ y $\left(\begin{array}{lll}0 & 1 / 2 & 1 / 2\end{array}\right)$. Esta estructura se denomina en la literatura como tipo L1o y para este caso se obtiene a partir de una transformación martensítica, enfriando rápidamente la aleación desde la fase $\varepsilon$ de alta temperatura, que presenta una estructura hexagonal. La fase $\tau$ presenta una anisotropía uniaxial a lo largo del eje [001] con $K=10^{6} \mathrm{~J} / \mathrm{m}^{3}$. Al agregar C o B esta fase se estabiliza y aumenta la habilidad de inducir el orden direccional a lo largo del eje fácil. Coercitividades de 2 a $3 \mathrm{kOe}$ y productos $(\mathrm{BH})_{\max }$ de $64 \mathrm{~kJ} / \mathrm{m}^{3}$ (8 MGOe) se han alcanzado para este imán de fase única.

\section{Aplicaciones de los IPs (USGS Mineral resources program, http://minerals.usgs.gov/.)}

Algunas aplicaciones donde se utilizan IPs, principalmente del tipo 2-14-1 son:

\begin{tabular}{ll}
\hline Categoría & Ejemplos de aplicación \\
\hline $\begin{array}{l}\text { Computadores y } \\
\text { automatización de } \\
\text { oficina }\end{array}$ & $\begin{array}{l}\text { Discos duros, motores de rotación de CD-ROM* } \\
\text { Motores de bobina de voz y pick-up } \\
\text { Motores de impresoras y fax }\end{array}$ \\
\hline $\begin{array}{l}\text { Electrónica de } \\
\text { consumo }\end{array}$ & $\begin{array}{l}\text { Videocámaras, reproductores DVD* } \\
\text { Altavoces, micrófonos y auriculares } \\
\text { Celulares }\end{array}$ \\
\hline Accesorios & $\begin{array}{l}\text { Motores de accesorios domésticos y aires } \\
\text { acondicionados } \\
\text { Bombas de agua } \\
\text { Sistemas de seguridad }\end{array}$ \\
\hline $\begin{array}{l}\text { Automatización en } \\
\text { fábricas }\end{array}$ & $\begin{array}{l}\text { Acoplamientos magnéticos y cojinetes } \\
\text { Zapatillas } \\
\text { Motores, servomotores y generadores }\end{array}$ \\
\hline Industria médica & $\begin{array}{l}\text { Imágenes por resonancia magnética } \\
\text { Instrumentos quirúrgicos e implantes médicos }\end{array}$ \\
\hline Automoción y & $\begin{array}{l}\text { Motores de encendido y motores dc sin escobillas } \\
\text { Ciclas eléctricas y vehículos híbridos y eléctricos* } \\
\text { Bombas de combustible eléctricas }\end{array}$ \\
\hline
\end{tabular}

\begin{tabular}{ll}
\hline Energías alternativas & $\begin{array}{l}\text { Sistemas de potencia de viento*, olas y mareas } \\
\text { Sistemas de generación de potencia } \\
\text { Sistemas de almacenamiento de energía }\end{array}$ \\
\hline \multirow{2}{*}{ Militar } & $\begin{array}{l}\text { Armas, proyectiles de precisión guiados } \\
\text { Vehículos, motos acuáticas, aviones } \\
\text { *Presentarán gran crecimiento en la próxima década }\end{array}$
\end{tabular}

Los IPs se pueden clasificar, de acuerdo a la función principal que desempeñen en una determinada aplicación, como:

- Transformar energía eléctrica en mecánica (motores, relés, altoparlantes, actuadores, sistemas micro electromecánicos (MEMS), etc.)

- Transformar energía mecánica en eléctrica (micrófonos, generadores, alternadores)

- Atracción o repulsión magnética para realizar trabajo (separadores, dispositivos de acoplamiento de rodamientos, unidades de torsión)

- Guía y control de carga eléctrica, plasmas y haces de electrones o iones)

- Almacenamiento de información.

\section{Futuro}

Al final de la década de los 90 la diferencia entre los precios de las materias primas (TRs) y de venta de IPs se hizo estrecha, y muchas de las empresas productoras fueron vendidas o se trasladaron a China (donde se produce 95\% de las TRs para IPs). Hoy en día para escoger los materiales para elaborar IPs se hace un balance entre precio y rendimiento. Las hexaferritas son más baratas y livianas que los imanes tipo $\mathrm{NdFeB}$ y por eso abarcan el $85 \%$ en peso del mercado global, sin embargo para pequeñas aplicaciones los $\mathrm{NdFeB}$ constituyen el $50 \%$ de este mercado. La proyección es que el mercado de los de NdFeB crecerá. Sin embargo desde el 2010 los precios del Nd han aumentado y presentaron un máximo del $800 \%$ en el 2012, y se cree que la demanda aumentará en un 200\%. Además a partir del 2009 China ha restringido la exportación de TRs, dejando la mayoría de la producción para el consumo interno. Esta situación ha creado la llamada crisis de las TRs.

En este contexto existe la necesidad de producir imanes con alto producto de energía, libres de TRs. Por eso actualmente se investigan 6 nuevos tipos de materiales magnéticos agrupados en dos categorías:

Categoría I: Desarrollar IPs nanocompuestos con: i) anisotropía de intercambio ferromagnético (Exchange spring magnets) y ii) anisotropía de intercambio antiferromagnético (Exchange biased magnets).

Categoría II: Desarrollar materiales magnéticos con estructura cristalina que posea alta anisotropía: iii) $\alpha^{\prime \prime}-\mathrm{Fe}_{16} \mathrm{~N}_{2}$; iv) FeNi tipo L10; v) $\mathrm{MnAl}(\mathrm{C}, \mathrm{B})$ tipo L1o; y vi) nanofases $\mathrm{Co}_{3} \mathrm{C} / \mathrm{Co}_{2} \mathrm{C}$. 


\section{Categoría I}

La Figura 7 muestra los ciclos de histéresis que se obtienen para sistemas nanocompuestos del tipo $i$ (Izq.) con granos ferromagnéticos de fase dura y blanda directamente acoplados, y de tipo ii (Der.) con granos ferromagnético y antiferromagnético directamente acoplados. En la parte superior de cada figura se muestra el comportamiento de las estructuras de espín en el cuadrante de desmagnetización de cada sistema. El ejemplo típico de sistemas $i$ es el de los imanes de $\mathrm{Nd}_{2} \mathrm{Fe}_{14} \mathrm{~B} / \alpha$-Fe o $\mathrm{Fe}_{2} \mathrm{~B}$ y de sistemas ii es el de $\mathrm{Co} / \mathrm{CoO}$. Se buscan sistemas magnéticos que puedan formar imanes permanentes nano-compuestos de estos dos tipos, libres de TRs. En los imanes del tipo $i$ la fase dura aporta la anisotropía y la alta $H_{c}$ y la blanda (y en menor grado la dura) aportan el alto $B_{s}\left(M_{s}\right)$, mientras que en los imanes de tipo ii el acople de intercambio hace que el ciclo se desplace a la izquierda, aumentando el $H_{c}$, y la fase ferromagnética aporta el $B_{s}\left(M_{s}\right)$. En este tipo de material la fase antiferomagnética no aporta a la magnetización.

\section{Categoría II}

\section{Tipo iii) Martensita ordenada y nitrogenada $\alpha^{\prime \prime}-\mathrm{Fe}_{16} \mathrm{~N}_{2}$}

A mediados de los 90 se desató gran actividad para tratar de explicar y comprobar el alto valor del momento magnético del Fe en el compuesto $\alpha^{\prime \prime}-\mathrm{Fe}_{16} \mathrm{~N}_{2}$ (del orden de $3.2 \mu_{B}$, cerca del $50 \%$ más que el del Fe puro que es $2.2 \mu_{B}$ ). Este fue reportado originalmente en forma de película por Kim \& Takahashi (1972). Fue comprobado por Takahashi, Mitsuoka, Komuro \& Sugita (1993) cuando se obtuvieron martensita ordenada. El material se obtuvo en bulto por templado de la fase austenita nitrogenada $\gamma-\mathrm{FeN}$, dando lugar a una martensita nitrogenada desordenada $\alpha^{\prime}$-FeN. Posteriormente fue tratada a baja temperatura, entre 127 y $202{ }^{\circ} \mathrm{C}$, para así obtener la fase martensita nitrogenada ordenada $\alpha^{\prime \prime}-\mathrm{Fe}_{16} \mathrm{~N}_{2}$. Ha sido difícil obtener esta fase en forma pura, pero en el 2011 http://energy.gov/sites/prod/ files/DOE_CMS2011_FINAL_Full.pdf se reportó esta fase, altamente orientada, reproducible, con un 91\% de pureza, y con magnetización mayor que la del Fe. Su estructura es tetragonal, pero aún no se consigue una anisotropía suficiente para considerarse lo suficientemente dura.

\section{Tipo L1o iv) FeNi y v) MnAl}

La mayoría de las aleaciones binarias de metales de transición presentan normalmente estructuras cúbicas magnéticas con baja anisotropía. Algunas pocas presentan la estructura tipo L1o, que es tetragonal ordenada con anisotropía uniaxial. Entre ellas están las aleaciones FePt y FePd que presentan alta magnetización y constante de anisotropía cristalina, con valores reportados de $(B H)_{\max }$ del orden de 20 MGOe (157 $\mathrm{kJ} / \mathrm{m}^{3}$ ), cuando se producen nanocompuestos con base en ellas. Debido a los altos costos del Pt y el Pd, solamente se utilizan en producir películas delgadas para grabado magnético y no para aplicaciones en bulto.
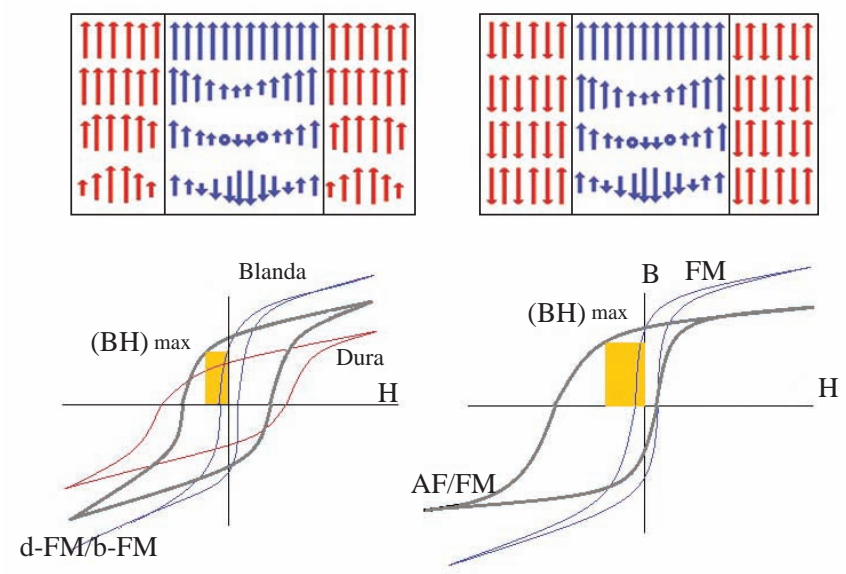

Figura 7. Comportamiento de la desmagnetización ( $2^{\circ}$ cuadrante) de sistemas de fases dura-blanda (Izq.) y de fases ferro-antiferro (Der.) y sus correspondientes ciclos de histéresis (Lewis \& Jiménez-Villacorta).

Como se dijo anteriormente la fase L1o se obtiene también para el caso del sistema $\mathrm{MnAl}$, por templado desde la fase hexagonal $\varepsilon$, de alta temperatura. Se reportan en la literatura (McCurrie, Rickman, Dunk \& Hawkridge (1978) y Fazakas, Varga \& Mazaleyrat (2007)) aleaciones FeMn y FeMn(C) producidas por melt-spinning y luego de tratamientos térmicos adecuados con valores de $H_{c}$ entre 1500 y 2000 Oe y productos $(B H)_{\max }$ entre 4 y 7 MGOe (37 y 56 kJ/m³) (Hoydick, Palmiere \& Soffa (1997) y Zeng, Baker, Cui \& Yan (2007). Por molienda mecánica se ha logrado reducir el tamaño de partícula de aleaciones FeMn(C) para obtener valores de $H_{c}$ de hasta 4000 Oe (Zeng, Baker \& Yan (2006). Este sistema es promisorio pero no alcanza todavía las características de las hexaferritas o los IPs de TRs.

Para caso del sistema FeNi la fase L1o se ha obtenido por bombardeo neutrónico de la fase equiatómica FeNi con estructura fcc desordenada, también llamada A1, donde los átomos tienen la misma probabilidad de encontrase en cualquier sitio. Para el sistema FeNi esta fase recibe el nombre de taenita y naturalmente se encuentra en meteoritos metálicos. En este tema todavía no se ha avanzado mucho por las grandes dificultades de obtener la fase L1o. Para el caso de meteoritos esta fase se ha encontrado pero se sabe que la tasa de enfriamiento esta entre 0.2 a $2000 \mathrm{~K} / \mathrm{año.}$

\section{Tipo vi) Nanofases $\mathrm{Co}_{3} \mathrm{C} / \mathrm{Co}_{2} \mathrm{C}$}

Recientemente se reportó que carburos de Co, sintetizados por el método químico de reducción de poliol, presentan $H_{c}$ mayores que $3.4 \mathrm{kOe}$ y $(\mathrm{BH})_{\max }$ mayores de $20 \mathrm{~kJ} / \mathrm{m}^{3}$ (2.5 MGOe) (Harris, Chen, Yang, Yoon, Chen, Geiler, Gao, Chinnasamy, Lewis, Vittoria, Carpenter, Carroll, Goswami, Willard, Kurihara, Gjoka \& Kalogirou (2010). Este material es compuesto de las nanofases $\mathrm{Co}_{3} \mathrm{C}$ 
y $\mathrm{Co}_{2} \mathrm{C}$ con morfología acicular y con $T_{c}$ de $237^{\circ} \mathrm{C}$. Los altos valores de $H_{c}$ pueden deberse a la interacción dipolar, la anisotropía de forma y posiblemente a la anisotropía de intercambio. El mejoramiento de estas propiedades y el entendimiento de los resultados es todavía un reto.

Finalmente vale la pena mencionar que el Grupo de Metalurgia Física y Teoría de Transiciones de Fase (GMTF) de la Universidad del Valle ha venido trabajando en aleaciones magnéticas blandas tipo FeAl (Pérez Alcázar \& Galvão da Silva (1987)), FeCo (Jiménez, Vélez, Zamora \& Pérez (2012)), FeNi (Restrepo, Pérez Alcázar \& Bohórquez (1997)), FeSi (Piamba \& Pérez Alcázar (2015)) y FeMnAl (Zamora, Pérez Alcázar, Bohórquez, Marco \& González (1997) y González, Pérez Alcázar, Zamora, Tabares \& Greneche (2002)). En el sistema ternario se han publicado diagramas de fase magnéticos experimentales que han sido comparados con los calculados teóricamente, obtenidos utilizando modelos de Ising y Heisemberg, usando técnicas estadísticas como el de la desigualdad de Bogoliuvob, los Grupos de Renormalización Campo Medio y el Método
Monte Carlo. A partir de 1988 el GMTF incursionó en el estudio de IPs tipo Nd-Fe-B, estudiando el efecto del $\mathrm{Nb}$, el Co y el Ni en sus propiedades magnéticas y estructurales. Con la aprobación a finales de 2015, del proyecto INAPEM (International Network on Applications of PErmanent Magnets) por parte de la Comunidad Económica Europea, el GMTF se encuentra ahora trabajando junto con colegas de España, Alemania, Grecia y Estados Unidos, en IPs tipo MnAl y tipo TRFe $_{12}(1-12)$.

\section{Conclusión}

En el estudio de IPs se buscan materiales magnéticos que presenten una nanoestructura compuesta de dos fases con acople ferromagnético: una fase dura con alta coercitividad, cuyo origen sea por la alta anisotropía magnética de su estructura cristalina o a la anisotropía de forma de sus granos; y otra fase blanda, con alta magnetización de saturación. Se busca además que los materiales que den lugar a estas dos fases sean de bajo costo, o sea que no contengan TRs o que la cantidad de estas sean muy bajas.

\section{Información suplementaria}

Figura 1S. Esquema representativo de los semiciclos de histéresis $B$ vs. $H$ (roja) y $M$ vs. $H$ (verde) (G. Hadjipanayis (2008)). http:// www.raccefyn.co/index.php/raccefyn/article/downloadSuppFile/ 361/1515

Figura 2S. Barra magnética sometida a campos paralelo y perpendicular al eje mayor (Izq.) y sus curvas de magnetización vs. campo aplicado (Der.). http://www.raccefyn.co/index.php/ raccefyn/article/downloadSuppFile/361/1516

Figura 3S. Campos de una barra magnetizada sin campo aplicado, (a) líneas de $H$, y (b) líneas de $B$. Los vectores de la mitad muestran las direcciones de $B, H_{d}$ y $M$ en el centro del imán. http://www. raccefyn.co/index.php/raccefyn/article/downloadSuppFile/ $361 / 1517$

Figura 4S. Curvas de desmagnetización de los imanes 1 y 2, junto con diferentes curvas de $B H$ constante y pendientes o permeabilidades. http://www.raccefyn.co/index.php/raccefyn/article/ downloadSuppFile/361/1518

Figura 5S. Curvas de magnetización para (Izq.) hierro y (Der.) níquel. http://www.raccefyn.co/index.php/raccefyn/article/download SuppFile/361/1519

Figura 6S. Estructura de dominios del Fe en el plano $\{100\}$. Se aplica un campo H en la dirección [110]. http://www.raccefyn.co/ index.php/raccefyn/article/downloadSuppFile/361/1520

Figura 7S. Curvas de magnetización para el cobalto. http:// www.raccefyn.co/index.php/raccefyn/article/downloadSuppFile/ $361 / 1521$

Figura 8S. Estructura de dominios de un sistema uniaxial. http:// www.raccefyn.co/index.php/raccefyn/article/downloadSuppFile/ 361/1522
Figura 9S. Material en forma de elipsoide prolata. http://www. raccefyn.co/index.php/raccefyn/article/downloadSuppFile/ $361 / 1523$

Figura 10S. Diagrama de fase seudobinario Fe-NiAl (De Vos (1969)). http://www.raccefyn.co/index.php/raccefyn/article/download SuppFile/361/1524

Figura 11S. Micrografía electrónica de un alnico enfriado en un campo horizontal (50.000X) (McCurrie (1982)). http://www. raccefyn.co/index.php/raccefyn/article/downloadSuppFile/ 361/1525

Figura 12S. Solidificación direccional de alnico a lo largo de [100]. http://www.raccefyn.co/index.php/raccefyn/article/download SuppFile/361/1526

Figura 13S. Curvas de B-H para algunos alnico anisotrópicos seleccionados; los productos $(\mathrm{BH})_{\max }$ se determinan de las hipérbolas a trazos, cada una con BH constante (McCurrie (1982)). http:// www.raccefyn.co/index.php/raccefyn/article/downloadSuppFile/ 361/1527

Figura 14S. Láminas hexagonales de hexaferrita de Ba obtenidas por precipitación (O’Handley). http://www.raccefyn.co/index.php/ raccefyn/article/downloadSuppFile/361/1528

Figura 15S. Micrografias de hexaferrita de Ba sinterizada, (Izq.) vista a lo largo del eje fácil, y (Der.) vista perpendicular al eje fácil (Smith y Wijn (1959)). http://www.raccefyn.co/index.php/ raccefyn/article/downloadSuppFile/361/1529

Figura 16S. Diagrama de fase de equilibrio del sistema SmCo en la región rica en Co (Den Broder \& Buschow (1977)). http:// www.raccefyn.co/index.php/raccefyn/article/downloadSuppFile/ 361/1530 
Figura 17S. Curva esquemática de magnetización y desmagnetización del $\mathrm{SmCo}_{5}$. http://www.raccefyn.co/index.php/raccefyn/ article/downloadSuppFile/361/1531

Figura 18S. Diferentes tipos de IPs para pequeñas aplicaciones: imanes de hexaferritas (Izq.), imanes de $\mathrm{Nd}_{2} \mathrm{Fe}_{14} \mathrm{~B}$ (Cent.), e imanes de alnico y cerámicos. http://www.raccefyn.co/index.php/raccefyn/ article/downloadSuppFile/361/1532

Figura 19S. Esquema de las características de los imanes nanocompuestos: acople entre fases duras y blandas cuando se invierte el campo (Izq.), ciclos de histéresis de las fases blanda y dura (Cent.), y ciclo de histéresis del material (Der.) (Hadjipanayis (2008)). http://www.raccefyn.co/index.php/raccefyn/article/download SuppFile/361/1533

Figura 20S. Diagrama de fases de equilibrio del sistema $\mathrm{MnAl}$ (Izq.) y estructura ordenada fct de la fase $\tau$ (estructura tipo $\mathrm{L1}_{0}$ ) (Lewis \& Jiménez-Villacorta). http://www.raccefyn.co/index. php/raccefyn/article/downloadSuppFile/361/1534

\section{Conflicto de intereses}

El autor declara no tener conflicto de intereses.

\section{Bibliografía}

Cullity B.D. \& Graham C.D. 2009. "Introduction to magnetic materials", Second Edition, a John Wiley \& Sons Publishing.

O’Handley R.C., 2000. "Modern magnetic materials: Principles and Applications", John Wiley \& Sons Publishing.

Jimenez-Villacorta F. \& Lewis L.H. 2014. “Advanced Permanent Magnetic Materials” en Nanomagnetism, Edition: http:// www.onecentralpress.com/ nanomagnetism/, Chapter: 7, Publisher: OCP Publishing Group. USGS Mineral resources program, http://minerals.usgs.gov/.

Lewis L.H. \& Jiménez-Villacorta F. 2011. "Perspectives on Permanent Magnetic Materials for Energy Conversion and Power Generation”, Metal. Mat. Trans. A, 44A, S1-S20. (https://www.google.com.co/search?ciclos+de+histéresis).

Hadjipanayis G. 2008. "Novel Materials for High Energy and Power Density”, University of Delaware, Energy Institute Symposium, March 17, Newark, DE.

Stoner E.C. 1945. “The demagnetizing factor for ellipsoids”, Phil. Mag., 36: 803-821.

Osborn, J. A. 1945. "Demagnetizing Factors of the General Ellipsoid”, Phys. Rev., 67: 351-357.

Akulov, N. 1936. “Zur Quantentheorie der Temperaturabhangigkeit der Magnetisie-rungskurve”, Z. Phys. 100: 197-202.

De Vos K.J., 1969. “Magnetism and Metallurgy”, Vol. 2, Academic Press.

McCurrie R.A. 1982. "Ferromagnetic Materials", Vol. 3, E. P. Wohlfarth, ed., North Holland, Amsterdam, p.107.

Smith J. \& Wijn H.P.J. 1959. "Ferrites”, Wiley, New York.

den Broder F.J.A. \& Buschow K.H.J. 1977, “Coercive force and stability of $\mathrm{SmCo}_{5}$ and $\mathrm{GdCo}_{5}$ ”, J. Less-Common Met. 29: 65-71.

Das B.N. \& Koon N.C. 1983, “Correlation between microstructure and coercivity of amorphous $\left(\mathrm{Fe}_{0.82} \mathrm{~B}_{0.18}\right)_{0.90} \mathrm{~Tb}_{0.05} \mathrm{La}_{0.05}$ alloy ribbons”, Met. Trans. 14A: 953-961.

Croat J.J. 1981, “Observation of large room-temperature coercivity in melt-spun $\mathrm{Nd}_{0.4} \mathrm{Fe}_{0.6}$ ”, Appl. Phys. Lett. 39:
357-358; 1981, “Crystallization and magnetic properties of melt-spun neodymium-iron alloys”, J. Magn. Magn. Mater. 24: $125-131$

Hadjipanayis G., Hazelton R.C., \& Lawless K.R. 1983, “New iron-rare-earth based permanent magnet materials”, Appl. Phys. Lett. 43: 797-798; 1984, "Cobalt-free permanent magnet materials based on iron-rare-earth alloys (invited)”, J. Appl. Phys. 55: 2073-2077.

Sagawa M., Fujimura S., Togawa M., \& Matsura Y., 1984 “New material for permanent magnets on a base of $\mathrm{Nd}$ and $\mathrm{Fe}$ (invited)”, J. Appl. Phys. 55: 2083-2087.

Croat J.J., Herbst J.F., Lee R.W., \& Pinlterton F.E. 1984, "Pr-Fe and Nd-Fe-based materials: A new class of highperformance permanent magnets (invited)”, J. Appl. Phys. 55: 2078-2082.

Herbst J.F., 1991. " $\mathrm{R}_{2} \mathrm{Fe}_{14} \mathrm{~B}$ materials: Intrinsic properties and technological aspects”, Rev. Mod. Phys. 63: 819-898.

Kim T.K. \& Takahashi M. 1972. "New Magnetic Material Having Ultrahigh Magnetic Moment”, Appl. Phys. Lett., 20: 492-494.

Takahashi H., Mitsuoka K., Komuro M., \& Sugita Y. 1993. J. Appl. Phys., 73: 6060-6062.

Conference on Critical Materials for a Clean Energy Future, October 4-5, 2011, Workshops hosted by the U.S. Department of Energy, Washington, DC, http://energy.gov/ sites/prod/files/DOE_CMS2011_FINAL_Full.pdf.

McCurrie R.A., Rickman J., Dunk P., \& Hawkridge D.G. 1978. "Dependence of the permanent magnet properties of $\mathrm{Mn}_{55} \mathrm{Al}_{45}$ on particle size”, IEEE Trans. Magn. 14: 682-684.

Fazakas E., Varga L.K., \& Mazaleyrat F. 2007. "Preparation of nanocrystalline $\mathrm{Mn}-\mathrm{Al}-\mathrm{C}$ magnets by melt spinning and subsequent heat treatments”, J. Alloy. Compd. 434-435: 611-613.

Hoydick D.P., Palmiere E.J., \& Soffa W.A. 1997. “Microstructural development in MnAl-base permanent magnet materials: New perspectives”, J. Appl. Phys. 81: 5624-5626.

Zeng Q., Baker I., Cui J.B., \& Yan Z.C. 2007. “Structural and magnetic properties of nanostructured $\mathrm{Mn}-\mathrm{Al}-\mathrm{C}$ magnetic materials”, J. Magn. Magn. Mater. 308: 214-216.

Zeng Q., Baker I., \& Yan Z. 2006. "Nanostructured Mn-Al permanent magnets produced by mechanical milling”, J. Appl. Phys. 99: 08E902. 
Harris V.G., Chen Y., Yang A., Yoon S., Chen Z., Geiler A.L., Gao J., Chinnasamy C.N., Lewis L.H., Vittoria C., Carpenter E.E., Carroll K.J., Goswami R., Willard M.A., Kurihara L., Gjoka M., \& Kalogirou O. 2010. "High coercivity cobalt carbide nanoparticles processed via polyol reaction: a new permanent magnet material”, J. Phys. D: Appl. Phys. 43: 165003.

Pérez Alcázar G.A. \& Galvão da Silva E., 1987. “Mossbauer effect study of magnetic properties of $\mathrm{Fe}_{1-\mathrm{q}} \mathrm{Al}_{\mathrm{q}}, 0<\mathrm{q}<0.5$, alloys in the disordered phase”, J. Phys. F: Met. Phys. 17: 2323-2335.

Piamba J. \& Pérez Alcázar G.A. 2015. "Effect of disorder on the structural and magnetic properties of the $\mathrm{Fe}_{50} \mathrm{Si}_{50}$ nanostructured system”, J. Alloys Comps. 643: S297-S301.

Jiménez Juana M., Vélez Germán Y., Zamora Ligia E., \& Pérez Germán A. 2012. "Estudio de las propiedades magnéticas y estructurales de aleaciones $\mathrm{Nd}_{16} \mathrm{Fe}_{76-\mathrm{x}} \mathrm{Ni}_{\mathrm{x}} \mathrm{B}_{8}$ con bajos contenidos de Ni”, Momento Rev. Fís. UNal. 44: 11-20.

Restrepo J., Pérez Alcázar G.A., \& Bohórquez A. 1997. "Description in a local model of the magnetic field distributions of $\mathrm{Fe}_{12-\mathrm{x}} \mathrm{Ni}_{\mathrm{x}}$ disordered alloys”, J. Appl. Phys. 81 (8): 4101-4103.

Zamora L.E., Pérez Alcázar G.A., Bohórquez A., Marco J.F., \& González J.M. 1997. "Magnetic properties of the $\mathrm{Fe}_{\mathrm{x}} \mathrm{Mn}_{0.70-\mathrm{x}} \mathrm{Al}_{0.30}(0.40<\mathrm{x}<0.58)$ alloy series”, J. Appl. Phys. 82 (12): 6165-6169.

González Claudia, Pérez Alcázar Germán A., Zamora Ligia E., Tabares Jesús A., \& Greneche Jean-Marc, 2002. "Magnetic properties of the $\mathrm{Fe}_{\mathrm{x}} \mathrm{Mn}_{0.600-\mathrm{x}} \mathrm{Al}_{0.400}, 0.200 \leq \mathrm{x}$ $\leq 0.600$, disordered alloy series", J. Phys.: Condens. Matter 14: 6531-6542. 